\title{
Numerical Calibration of a Low-Speed sUAS Flush Air Data System
}

\author{
Roger J. LAUrence III ${ }^{\mathrm{a}}$ AND BRIAN M. ARgrow \\ Smead Aerospace Engineering Sciences, University of Colorado Boulder, Boulder, Colorado
}

(Manuscript received 15 November 2018, in final form 17 April 2019)

\begin{abstract}
A method using computational fluid dynamics to numerically calibrate a flush air data system is presented. A small unmanned aircraft system (sUAS) has been equipped with a flush air data system and experimentally tested. The flush air data system uses computational fluid dynamics to train neural networks and is validated using the in-flight data that were previously collected. Results of the flight validation are presented, along with ways to improve the accuracy of the system. Several different calibration approaches are presented and compared with each other. The best-case results with the in-flight calibration are $0.59^{\circ}$ and $0.66^{\circ}$ for angle of attack and sideslip, respectively, whereas the best-case results when calibrated with computational fluid dynamics data are $0.78^{\circ}$ and $0.90^{\circ}$. It is also possible to estimate other air data parameters, such as dynamic pressure, static pressure, and density, with neural networks, but the direct calculation is more accurate. Calibrating the system numerically, such as with the use of computational fluid dynamics, removes the need for any calibration flights. Although not as accurate as the in-flight calibration, numerical calibration is possible and can save the user time and expense.
\end{abstract}

\section{Introduction}

Small unmanned aircraft systems (sUAS) have been used for meteorological measurements, including wind sensing (Kocer et al. 2011; Wildmann et al. 2014a). An overview of sUAS wind measurement techniques can be found in Elston et al. (2015). A common method of making wind measurements involves the use of multihole probes (MHP). These probes are similar to pitotstatic probes for airspeed, but with additional ports to resolve the full 3D probe-relative wind (Telionis et al. 2009). Small unmanned aircraft systems equipped with an MHP have proven effective for wind measurements (van den Kroonenberg et al. 2008; Wildmann et al. 2014b; Houston et al. 2016). Because the MHP must be mounted externally on the sUAS airframe (see Fig. 1) they are often susceptible to damage, particularly during landing, and the cost of a commercially manufactured MHP may be an order of magnitude greater than that of the sUAS.

Current affiliation: Ball Aerospace \& Technologies Corp., Boulder, Colorado.

Corresponding author: Roger Laurence, roger.laurenceiii@ colorado.edu
Mayer and Hattenberger (2012) and Lie and GebreEgziabher (2013) describe wind estimation approaches that employ the onboard navigation sensors, and Callegari et al. (2006) and Fei et al. (2007) describe wind sensing from sUAS that do not require obtrusive external hardware. Another method of wind sensing that is free of external hardware, and that has been studied extensively, is the flush air data system (FADS). A FADS operates similarly to an MHP (fusing pressure measurements from multiple ports), but instead of the ports being integrated into an externally mounted probe, the pressure ports for the FADS are integrated flush with the surface of the airframe. The first FADS was deployed in the 1960s on the X-15 rocket plane (Cary and Keener 1965), and FADS has since been implemented on aircraft of various sizes, from large aircraft (Siemers et al. 1983; Larson et al. 1987; Kalogiros and Wang 2002) to small unmanned aircraft (Samy et al. 2010; Quindlen and Langelaan 2013).

Multiple approaches to FADS calibration have been demonstrated, including radar tracking or dynamic maneuvers (Haering 1995), wind tunnel experiments (Quindlen and Langelaan 2013), or by merging multiple sources, such as inertial measurements, radar tracking, and weather balloon soundings (Whitmore et al. 1996). Calibration based on radar tracking is likely not available for typical sUAS operators, and wind tunnel calibration 


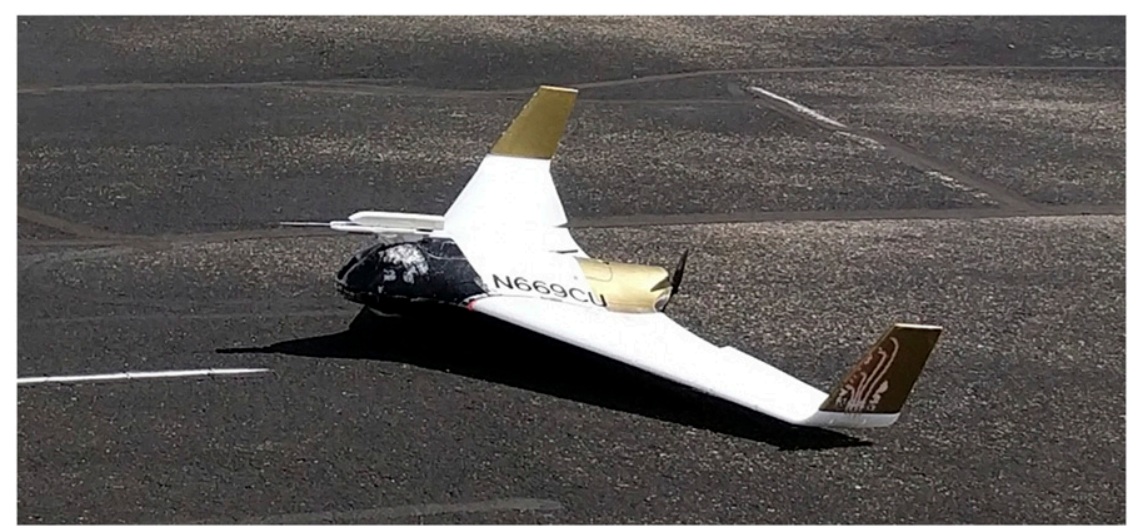

FIG. 1. The X-8 Skywalker sUAS with Aeroprobe five-hole MHP and FADS.

requires both access to a wind tunnel and that the airframe, with the integrated wind-measuring sensors, be small enough to fit into the tunnel. A quick search of the sUAS available will show a wide variety of shapes and sizes, many of which will not fit in most wind tunnels.

Laurence and Argrow (2018) describe the calibration of a FADS using an in-flight comparison to a reference MHP that was mounted on the sUAS airframe. This approach could decrease the per-instrument cost of calibration, especially when calibrating multiple aircraft, as one MHP can be used for all the aircraft. The MHP is only required for calibration flights; once calibrated, future flights are performed without the MHP.

The next logical goal is to eliminate experimental calibration entirely. Doing so could reduce costs and development time. It is possible to calibrate the FADS with analytical solutions, but this requires simple geometries (Cobleigh et al. 1999). Early FADS employed semiempirical approaches in which a theoretical model is corrected with empirical data (Pruett et al. 1983; Whitmore 1991). A similar approach fused numerical data with noisy and sparse empirical data to smooth the response (Srivastava et al. 2010). The first apparent calibration to do away with experimental data entirely involved computational fluid dynamics (Johnston et al. 1998). Similar to early approaches, a correction factor is applied to modified Newtonian flow theory; however, this correction factor is determined through computational fluid dynamics (CFD) and not empirical data. Flight results showed that the system generally satisfied flight control requirements, and that experimental calibration is not mandatory. Interestingly, the Mars Science Laboratory had a FADS built into the heat shield, and it was also calibrated with CFD data (Karlgaard et al. 2014). Both Johnston et al. (1998) and Karlgaard et al. (2014) show that a FADS can be successfully calibrated with CFD, although both systems only operated at hypersonic or supersonic flight speeds. Razzaqi et al.
(2012) also calibrated a FADS with CFD for hypersonic speeds, although the system was not validated with flight data.

Evidently, FADS calibration with CFD has not been implemented with low-speed small UAS. CFD can be used to predict the pressure distribution across arbitrary shapes, thus allowing the user to work with any sUAS. This approach is particularly useful when CFD has already been used to determine the port locations for the FADS (Samy et al. 2010; Laurence and Argrow 2018) so that the pressure distribution on the airframe is therefore predicted in advance.

This paper focuses on the calibration of the X-8 Skywalker sUAS with numerical data from CFD and validation of the system using flight data collected previously (Laurence and Argrow 2018). Section 2 presents updated CFD simulations that more closely match the flight conditions, compared to the original CFD simulations discussed in Laurence and Argrow (2018), along with how neural networks were used to estimate the angles of attack and sideslip from the pressure measurements. Section 3 discusses the results from multiple approaches to the CFD calibration of the FADS, and a general discussion and possible improvements are presented in section 4 . Conclusions are then presented in section 5 .

\section{CFD simulations}

CFD was used previously to determine the location of the pressure ports for the FADS on the X-8 Skywalker (Laurence and Argrow 2018). The original simulation parameters are presented in Table 1. The altitude $h$ is referenced against mean sea level (MSL) and is used with the U.S. Standard Atmosphere, 1976 (COESA 1976) to calculate the freestream static pressure $P_{\infty}$, freestream density $\rho_{\infty}$, and freestream temperature $T_{\infty}$. After flight tests, it was noticed that the expected flow 
TABLE 1. CFD simulation parameters.

\begin{tabular}{lc}
\hline \hline Parameter & \multicolumn{1}{c}{ Range of values } \\
\hline & Original simulations \\
$\alpha$ & {$\left[-4^{\circ},-2^{\circ}, 0^{\circ}, 2^{\circ}, 4^{\circ}, 6^{\circ}, 8^{\circ}, 10^{\circ}, 12^{\circ}\right]$} \\
$\beta$ & {$\left[-6^{\circ},-4.5^{\circ},-3^{\circ},-1.5^{\circ}, 0^{\circ}, 1.5^{\circ}, 3^{\circ}, 4.5^{\circ}, 6^{\circ}\right]$} \\
$V_{\infty}$ & {$[14,17,20] \mathrm{m} \mathrm{s}^{-1}$} \\
$h$ & {$[1830,2440] \mathrm{m}(\mathrm{MSL})$} \\
$P_{\infty}$ & $81200 \mathrm{~Pa}(h=1830 \mathrm{~m})$ \\
$P_{\infty}$ & $75250 \mathrm{~Pa}(h=2440 \mathrm{~m})$ \\
$\rho_{\infty}$ & $1.02 \mathrm{~kg} \mathrm{~m}^{-3}(h=1830 \mathrm{~m})$ \\
$\rho_{\infty}$ & $0.96 \mathrm{~kg} \mathrm{~m}^{-3}(h=2440 \mathrm{~m})$ \\
& \\
$\alpha$ & $\mathrm{Updated} \mathrm{simulations}^{\circ}$ \\
$\beta$ & {$\left[1^{\circ}, 2^{\circ}, 3^{\circ}, 4^{\circ}, 5^{\circ}, 6^{\circ}, 7^{\circ}\right]$} \\
$V_{\infty}$ & {$\left[-6^{\circ},-4^{\circ},-2^{\circ}, 0^{\circ}, 2^{\circ}, 4^{\circ}, 6^{\circ}\right]$} \\
$P_{\infty}$ & {$[18,22,26] \mathrm{m} \mathrm{s}^{-1}$} \\
$\rho_{\infty}$ & {$[82500,83500] \mathrm{Pa}^{-3}$} \\
\hline
\end{tabular}

conditions used for the CFD simulations differed from the actual in-flight flow conditions measured by the reference MHP during flight testing. As such, the CFD simulations have been updated to more accurately match the true flight conditions experienced by the Skywalker.

The inflight angles of attack $\alpha$ and sideslip $\beta$, as measured by the reference Aeroprobe Corp. five-hole MHP, are shown in Fig. 2. The calibration subset contains 24262 data points, and the validation subset contains 3143 data points. The highest concentration of data occurs around $\alpha=3^{\circ}$ and $\beta=1^{\circ}$, and $90 \%$ of the measurements are within $1^{\circ} \leq \alpha \leq 7^{\circ}$ and $-6^{\circ} \leq \beta \leq 6^{\circ}$. Although not shown, $95 \%$ of the true airspeed measurements are between 18 and $26 \mathrm{~m} \mathrm{~s}^{-1}, 89 \%$ of the static pressure measurements are between 82500 and $83500 \mathrm{~Pa}$, and $84 \%$ of the density measurements are between 0.97 and $0.99 \mathrm{~kg} \mathrm{~m}^{-3}$. From the flight data, the flow conditions used for CFD simulations have been updated, and they are presented in Table 1.

The CFD simulations were performed with the commercial software STAR-CCM+ (Siemens 2017). A polyhedral volume mesh was used, with approximately 110000 cells on the surface of the Skywalker model. The cell diameters on the Skywalker surface ranged from a minimum of $1.5 \mathrm{~mm}$ to a maximum of $6.5 \mathrm{~mm}$. The volume mesh is presented in Fig. 3. Flow was steady state and set as an ideal gas. The $k-\varepsilon$ turbulence model (Mohammadi and Pironneau 1994) was also incorporated into the simulations. Because the pressure ports of the FADS are located on the leading edges, there are no adverse pressure gradients or separated flow in the regions of interest; as such, the $k-\varepsilon$ model is a suitable choice. The pressure port locations are displayed in Fig. 4; 16 locations are displayed, but because of hardware issues during installation, suitable flight data for

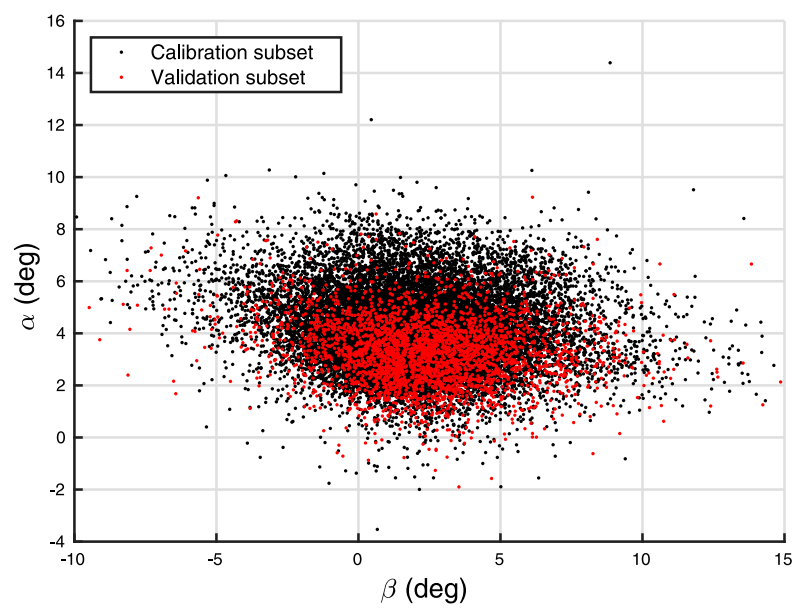

FIG. 2. MHP-measured $\alpha$ and $\beta$ for the calibration (black) and validation (red) subsets of the flight data. The full flight dataset is the union of these two datasets.

locations 2, 5, 11, and 13 are lacking, and these locations are excluded from the analysis. The two ports $P_{\mathrm{S} 1}$ and $P_{\mathrm{S} 2}$ dedicated to the measurement of the freestream static pressure and the single port $P_{t}$ for stagnation/total pressure are also displayed. Additionally, the propeller is excluded from the simulations, as is any other external hardware, and the control surfaces are in the neutral position. Note also that the cell grid and physics models are the same between the original and updated simulations; only the flow conditions are different. Readers are referred to Laurence and Argrow (2018) for a discussion of how the pressure ports were chosen, along with an explanation as to why certain pressure sensors do not have usable flight data.

\section{Calibration}

Neural networks have been used extensively with flush air data systems (Laurence and Argrow 2018; Quindlen and Langelaan 2013; Samy et al. 2010; Crowther and Lamont 2000; Rohloff 1998). The defining equation for a multilayer feed-forward neural network is

$$
\mathbf{a}_{i}=f_{i}\left(\mathbf{W}_{i} \mathbf{p}_{i}+\mathbf{b}_{i}\right),
$$

where $\mathbf{p}_{i}$ is the input into the $i$ th layer, $\mathbf{b}_{i}$ is the bias vector, $\mathbf{W}_{i}$ is the weight matrix, $f_{i}$ is the transfer function, and $\mathbf{a}_{i}$ is the output from the $i$ th layer. It has been shown that only a single hidden layer is required to approximate any arbitrary function (Hornik et al. 1989); because of this, all the networks presented in the paper will have a single hidden layer (layer 1) and a single output layer (layer 2). The hidden-layer transfer function $f_{1}$ is the hyperbolic tangent sigmoid function "tansig" (MathWorks 2018b), and the output layer $f_{2}$ uses the 


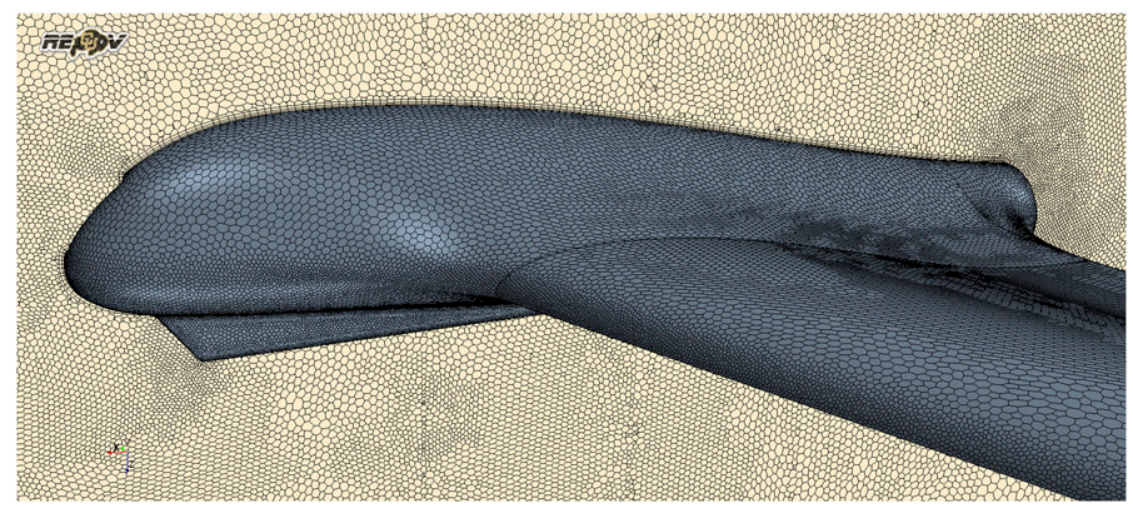

FIG. 3. Surface mesh of the Skywalker, along with a plane section through the airframe centerline.

linear function "purelin" (MathWorks 2018a). The inputs $\mathbf{p}_{1}$ for networks presented in this paper will vary depending on the calibration approach and will be detailed in the relevant sections in section 3 . The outputs $\mathbf{a}_{2}$ will include the angle of attack $\alpha$ and angle of sideslip $\beta$ but, depending on the calibration approach, may also include other air data parameters.

The weights and biases are determined during the training of the network. There are multiple methods available for training, but this paper will primarily focus on Bayesian regularization (Foresee and Hagan 1997; MacKay 1992). Bayesian regularization is a form of supervised learning, where the weights and biases are adjusted based on the error between the true outputs (measured by a reference instrument) and the outputs estimated by the network from the corresponding set of inputs. For example, with the in-flight calibration, the network produces an estimate of $\alpha$ and $\beta$ from in-flight measurements of $\Delta P$, indicated airspeed, $P_{\infty}$, and $\rho_{\infty} ; \Delta P$ is defined as the subtraction of $P_{\infty}$ from the absolute pressure measurement (i.e., the pressure directly measured by the sensors). The weights and biases are then adjusted based on the error between the estimate of $\alpha$ and $\beta$ from the network and the $\alpha$ and $\beta$ measurements from the reference MHP. This adjustment continues until certain training criteria are met, after which the weights and biases are finalized. The process is identical for the CFD calibration case, except the input and output data come from the simulation dataset and not from flight data. ${ }^{1}$

The initial weights and biases are set with the NguyenWidrow algorithm (Nguyen and Widrow 1990; MathWorks

\footnotetext{
${ }^{1}$ It is important to note that all methods are validated with the in-flight data; CFD simulation data are only used during the training of the networks and not during the validation.
}

2017). There is some randomness in this method of initializing the weights. In addition, of the portion of the dataset potentially used for training, only a random $85 \%$ are actually used for training; the remaining $15 \%$ serve as a check. Because of the randomness mentioned, simply reinitializing a network can lead to different network accuracies. All calibration methods therefore train an ensemble of 250 networks, but results are only presented using the single best network from the ensemble (how the best network is chosen is detailed in section 3).

With some technical aspects of neural networks explained, the general workflow will now be presented (see the flowchart in Fig. 5). There are two phases dealing with the neural networks: the training phase, and the validation phase. Training is the process of determining the network weights that will minimize the error between the training inputs and training targets. The dataset the training inputs come from is determined by the particular calibration approach. As explained later, the in-flight calibration uses the calibration subset of the flight dataset, whereas CFD-1 uses the original CFD dataset. The training targets come from the same dataset as the training inputs. The training inputs are values that the FADS is measuring, and are a combination of $\Delta P$, indicated airspeed, $P_{\infty}$, $\rho_{\infty}, q_{\infty}, P_{\mathrm{S} 1}, P_{\mathrm{S} 2}$, and $P_{t}$, whereas the training targets are the values of what the FADS is trying to estimate (and come from the reference MHP or CFD simulation data) and are a combination of $\alpha, \beta, q_{\infty}, P_{\infty}$, and $\rho_{\infty}$ The chosen training method adjusts the initial weights to minimize the error between the training targets and the network output based on the training inputs. Training ends after some stopping condition is met, such as an error threshold or number of iterations. Validation inputs, which are the same inputs as the training inputs, but from a different dataset, are input into the final 


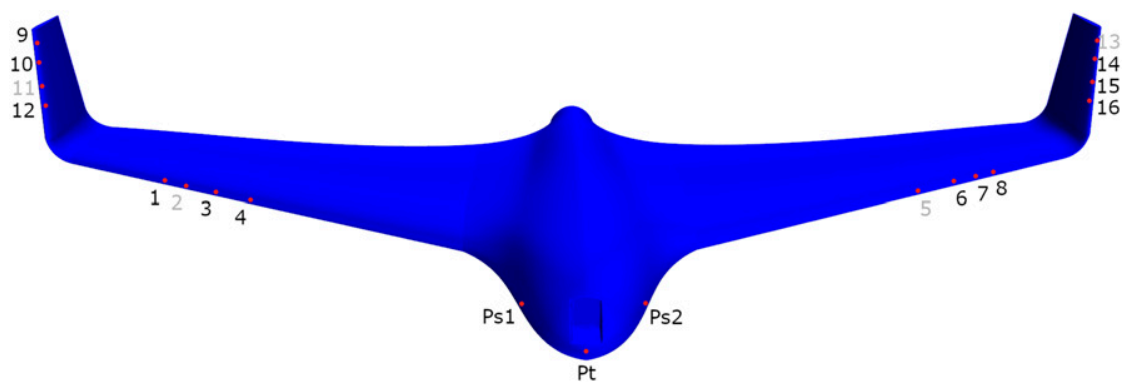

FIG. 4. Pressure port locations. Location numbers that have been grayed out $(2,5,11$, and 13$)$ are excluded from the analysis.

network to produce the validation outputs. It should also be noted that validation will only use the flight dataset. These outputs can then be compared with the reference MHP measurements from the same dataset. This is how the performance of the FADS will be characterized.

\section{Results}

\section{a. Original in-flight calibration}

The in-flight calibration was originally presented and detailed in Laurence and Argrow (2018); a brief review and relevant results are presented here. The Skywalker UAS, with the installed FADS and reference MHP (Aeroprobe 2016), was flown on 22 March 2017, near Boulder, Colorado. The testing consisted of two flights, each lasting approximately $40 \mathrm{~min}$. As previously mentioned in section 2 , the full flight dataset was split into two subsets: calibration and validation, with the calibration subset being approximately $90 \%$ of the total flight data. Please refer back to Fig. 2 to see the difference between the calibration and validation datasets. The calibration portion of the flight was designed such that the aircraft would experience a wide range of $\alpha, \beta$, and $V_{\infty}$, while the validation portion consisted of typical flight maneuvers (ascending/descending orbits and transects). Pressure at the FADS ports was measured with MS8607 (TE Connectivity 2015) pressure, temperature, and humidity sensors. These sensors were chosen for their low cost $(\sim \$ 10$ per sensor $)$ and high resolution with a small form factor. The sensors have a resolution of $1.6 \mathrm{~Pa}$, a relative accuracy ${ }^{2}$ of $10 \mathrm{~Pa}$, and sensor noise $\mathrm{e}^{3}$ of approximately $2 \mathrm{~Pa}$ (1 standard deviation).

\footnotetext{
${ }^{2}$ Accuracy is relative to the previous measurement as opposed to absolute accuracy.

${ }^{3}$ Sensor noise is determined using preflight data in a motor-off configuration.
}

As discussed previously, the flow angles $\alpha$ and $\beta$ are estimated with neural networks. Airspeed, on the other hand, is directly calculated with the standard pitot equation:

$$
V_{\infty}=\left(2 q_{\infty} / \rho_{\infty}\right)^{1 / 2},
$$

where $q_{\infty}$ is the dynamic pressure and is simply the difference between the stagnation (or total) pressure $P_{t}$ and the freestream static pressure $P_{\infty}$ :

$$
q_{\infty}=P_{t}-P_{\infty}
$$

$P_{t}$ is measured at the stagnation port on the nose of the Skywalker, and $P_{\infty}$ is the average of the two static port locations on the side of the nose of the Skywalker. The density is represented by $\rho_{\infty}$ and is computed using the ideal gas law. When $\rho_{\infty}$ is set to the reference sea level density $\rho_{0}\left(1.225 \mathrm{~kg} \mathrm{~m}^{-3}\right), V_{\infty}$ is termed indicated airspeed (IAS); when the true value of $\rho_{\infty}$ is used, it is termed true airspeed (TAS). Because only a simple analytical equation is required to calculate airspeed, airspeed results are not the focus of this paper, and they will only be briefly discussed in a few sections of the paper. Results will instead concentrate on $\alpha$ and $\beta$.

Errors $\varepsilon_{\alpha}$ and $\varepsilon_{\beta}$ with the FADS estimates are defined as

$$
\varepsilon_{i}=\hat{Y}_{i}-Y_{i},
$$

where $\hat{Y}_{i}$ is the estimate from the FADS based on the $i$ th measurement and $Y_{i}$ is the value from the reference MHP. A positive error means the FADS is overestimating the value compared to the MHP. In addition to the simple error statistics, such as the mean error $\mu$ and standard deviation of the error $\sigma$, another useful error metric is the root-mean-square error (RMSE):

$$
\operatorname{RMSE}=\left[\frac{1}{n} \sum_{i=1}^{n}\left(\hat{Y}_{i}-Y_{i}\right)^{2}\right]^{1 / 2}=\left(\mu^{2}+\sigma^{2}\right)^{1 / 2},
$$




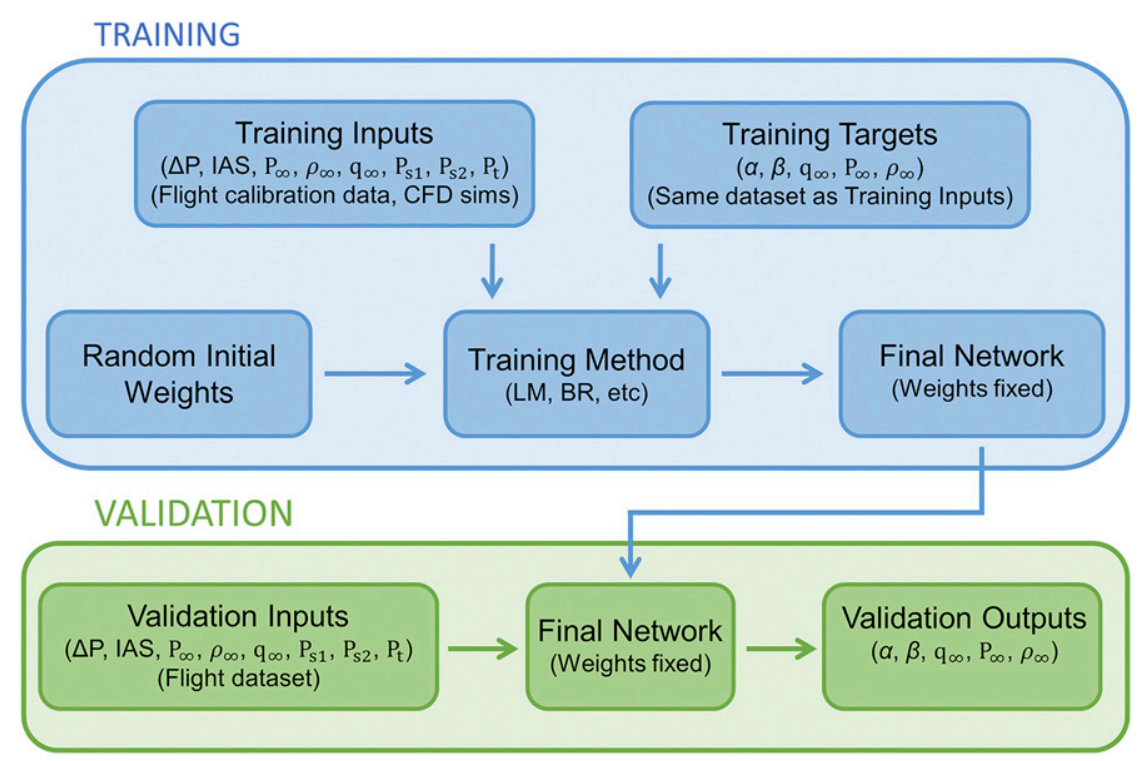

FIG. 5. Flowchart explaining the use of neural networks.

with $n$ representing the number of measurements in the dataset. When the errors are unbiased, ${ }^{4}$ the RMSE is equal to the standard deviation of the errors.

Network inputs are the differential pressures from the 12 ports $\Delta P$, IAS, $P_{\infty}$, and $\rho_{\infty}$, and the outputs are $\alpha$ and $\beta$. As described in Laurence and Argrow (2018), the in-flight calibration network was chosen by sorting all of the networks based on the norm of the RMSE:

$$
\begin{aligned}
\|\mathrm{RMSE}\| & =\left\|\left(\mathrm{RMSE}_{\alpha}, \mathrm{RMSE}_{\beta}\right)\right\| \\
& =\left(\mathrm{RMSE}_{\alpha}^{2}+\mathrm{RMSE}_{\beta}^{2}\right)^{1 / 2} .
\end{aligned}
$$

Several thousand networks were trained, each with a hidden-layer size of nine nodes, with the best network selected by minimizing $\|$ RMSE $\|$. Simple statistics of the FADS errors using this network are presented in Table 2. As a reminder, this network was trained with the calibration subset of the flight data. It is immediately clear that the errors are unbiased and that the standard deviation in $\varepsilon_{\beta}$ is larger than that in $\varepsilon_{\alpha}$. The authors hypothesize that the cause of this discrepancy might be due to the increased sensitivity of the airframe to $\alpha$ relative to $\beta$ and to the flexing in the outboard portions of the wings. Unsurprisingly, the FADS performs better with the calibration dataset when compared with the validation dataset, but only slightly better. It is important to note that for the results presented the MHP measurements are taken to be the

\footnotetext{
${ }^{4}$ For an unbiased network, $\mu=0$.
}

truth. It is known that the MHP will have its own errors, but it is one of the best reference instruments available to sUAS. ${ }^{5}$

While RMSE is useful for sorting the networks, because of the influence of mean errors the RMSE value is not necessarily the best metric. If the mean errors are known, results can be improved by subtracting the mean errors out. For example, consider two networks with identical RMSE values, one network that is unbiased and a second that is heavily biased. If the bias in the second network is known and removed, it will improve the RMSE. It is important to note that this paper is not focused on choosing the optimal network; instead this paper is focused on how well the FADS can be calibrated with CFD, compared to the in-flight calibration that was originally implemented. Because biases can be removed, the results will focus on the standard deviation of errors, which cannot be improved without changing the network.

A hidden-layer size of nine nodes was chosen corresponding to the size at which the RMSE norm was lowest; however, this is not necessarily the best size for $\alpha$ or $\beta$ when viewing the standard deviation of errors. To determine the lowest $\sigma_{\alpha}$ and $\sigma_{\beta}$, more networks were trained, with hidden-layer sizes ranging from 2 to 15 nodes, and an ensemble of 250 networks per hiddenlayer size (for a total of 3500 networks). The network

\footnotetext{
${ }^{5}$ Errors in the MHP measurements from upwash produced by the wing and local flow field distortions from the fuselage are mitigated using the standard practice of placing the air data probe as far upstream of the airframe as is practicable.
} 
TABLE 2. Error statistics; all three networks have nine nodes in the hidden layer and were sorted on the basis of the norm of the RMSE.

\begin{tabular}{lrrrrr}
\hline \hline & & & $\begin{array}{c}\text { Calibration } \\
\text { dataset }\end{array}$ & $\begin{array}{r}\text { Validation } \\
\text { dataset }\end{array}$ & $\begin{array}{r}\text { Full } \\
\text { dataset }\end{array}$ \\
\hline In-flight calibration & $\mu$ & $\alpha$ & $0.00^{\circ}$ & $0.02^{\circ}$ & $0.00^{\circ}$ \\
& & $\beta$ & $0.00^{\circ}$ & $0.00^{\circ}$ & $0.00^{\circ}$ \\
& $\sigma$ & $\alpha$ & $0.56^{\circ}$ & $0.65^{\circ}$ & $0.57^{\circ}$ \\
CFD-1 & & $\beta$ & $0.79^{\circ}$ & $0.87^{\circ}$ & $0.80^{\circ}$ \\
& $\mu$ & $\alpha$ & $-1.30^{\circ}$ & $-0.38^{\circ}$ & $-1.20^{\circ}$ \\
& & $\beta$ & $-0.42^{\circ}$ & $-0.91^{\circ}$ & $-0.47^{\circ}$ \\
CFD-2 & $\sigma$ & $\alpha$ & $1.25^{\circ}$ & $1.35^{\circ}$ & $1.29^{\circ}$ \\
& & $\beta$ & $1.79^{\circ}$ & $1.97^{\circ}$ & $1.82^{\circ}$ \\
& $\mu$ & $\alpha$ & $-0.32^{\circ}$ & $0.10^{\circ}$ & $-0.27^{\circ}$ \\
& & $\beta$ & $-0.72^{\circ}$ & $-1.10^{\circ}$ & $-0.77^{\circ}$ \\
& $\sigma$ & $\alpha$ & $1.00^{\circ}$ & $1.20^{\circ}$ & $1.04^{\circ}$ \\
& & $\beta$ & $1.58^{\circ}$ & $1.81^{\circ}$ & $1.61^{\circ}$ \\
\hline
\end{tabular}

inputs are the same as described previously, as are the outputs. Results using the full dataset are broken up by hidden-layer size and presented in Fig. 6. Per the MATLAB boxplot function, the central mark represents the median and the left and right edges of the box indicate the 25 th and 75 th percentiles, respectively. The whiskers include all points that are not considered to be outliers, and the outliers are shown by plus signs (defined as greater than approximately $\pm 2.7 \sigma$ ). From Fig. 6 , it is clear that the majority of the networks have $\alpha$ error standard deviations of less than $0.6^{\circ}$ (not shown, but the same can be said for $\beta$ with $0.9^{\circ}$ ). It is also clear that the hidden-layer size has only a small effect on the accuracy of the system. Because of this near independence to hidden-layer size, it is unsurprising that the original results from the in-flight calibration (Table 2) are nearly identical to the best-case results shown in Fig. 6. Results showing all 3500 networks lumped together are shown in Fig. 7.

Multiple calibration approaches using the CFD simulation data are presented in the following sections. Each approach utilizes the CFD data differently for calibration, but all approaches are validated using the same in-flight data, with results presented in their respective sections. A concise summary of the different calibration methods is given in Table 3 .

\section{b. CFD-1 calibration}

The previous section outlined the in-flight calibration for estimating $\alpha$ and $\beta$. This method trained neural networks on the calibration subset of the in-flight data, and then assessed the accuracy of the system using the validation subset. The first method that calibrates the system exclusively with CFD data is CFD-1. This method uses the original CFD data, since that dataset was available prior to the flight testing.
During the network training, instead of using the calibration dataset from flight testing, the original CFD simulations are used. This dataset has 486 data points (as compared with the 24262 data points in the flight calibration dataset). The simulation parameters can be found in Table 1 . The $\Delta P$ measurements come from the CFD cells that correspond to the pressure sensor locations, ${ }^{6}$ whereas $q_{\infty}, P_{\infty}$, and $\rho_{\infty}$ are set boundary conditions for the simulations. IAS, used as an input for in-flight calibration, has been replaced by $q_{\infty}$. This does not have an effect on the network because IAS is proportional to $q_{\infty}$. Outputs for the network are $\alpha$ and $\beta$, which are also set boundary conditions for the simulations.

Upon completion of training the networks with the CFD data, the networks were validated using the inflight dataset. For validation, $\Delta P, q_{\infty}, P_{\infty}$, and $\rho_{\infty}$ all come from actual measurements from the FADS, while the reference $\alpha$ and $\beta$ measurements came from the MHP. Like the original in-flight calibration results, the network is kept to nine nodes, and the best network is chosen by minimizing $\|\mathrm{RMSE}\|$; these results are displayed in Table 2. One of the most notable differences is that the CFD-1 results are biased. The errors are also larger, with both $\sigma_{\alpha}$ and $\sigma_{\beta}$ being 2 times as large relative to the in-flight calibration results, for the validation dataset. As before, results using the validation and calibration datasets are comparable. The results presented so far show that, even though CFD calibration is less accurate, it still shows promise. However, similar to before, it is unknown whether a network with nine nodes and sorted by $\|$ RMSE $\|$ actually produces the best results. More networks were trained, with hiddenlayer sizes ranging from 2 to 15 nodes, also with 250 networks per hidden-layer size. Figures similar to Fig. 6 are not presented for CFD-1, but the results from the 3500 trained networks using the full dataset are presented in Fig. 7. The best cases for $\sigma_{\alpha}$ and $\sigma_{\beta}$ are approximately $0.75^{\circ}$ and $1.30^{\circ}$, respectively, which is an improvement over the values of $1.25^{\circ}$ and $1.79^{\circ}$ when using nine nodes and sorting by $\|$ RMSE $\|$. Results using just the validation dataset (not shown) are very similar to full dataset results.

It is important to determine whether the best-case results are actually outliers or not. A simple way of doing this is to focus not only on the absolute best case, but also networks that are not the absolute best. For example, the best performance achieved for $\sigma_{\alpha}$ with the

\footnotetext{
${ }^{6}$ Pressure sensors are located slightly set back from the leading edge, with three on each winglet and three on each wing. See Fig. 4 for the port locations.
} 


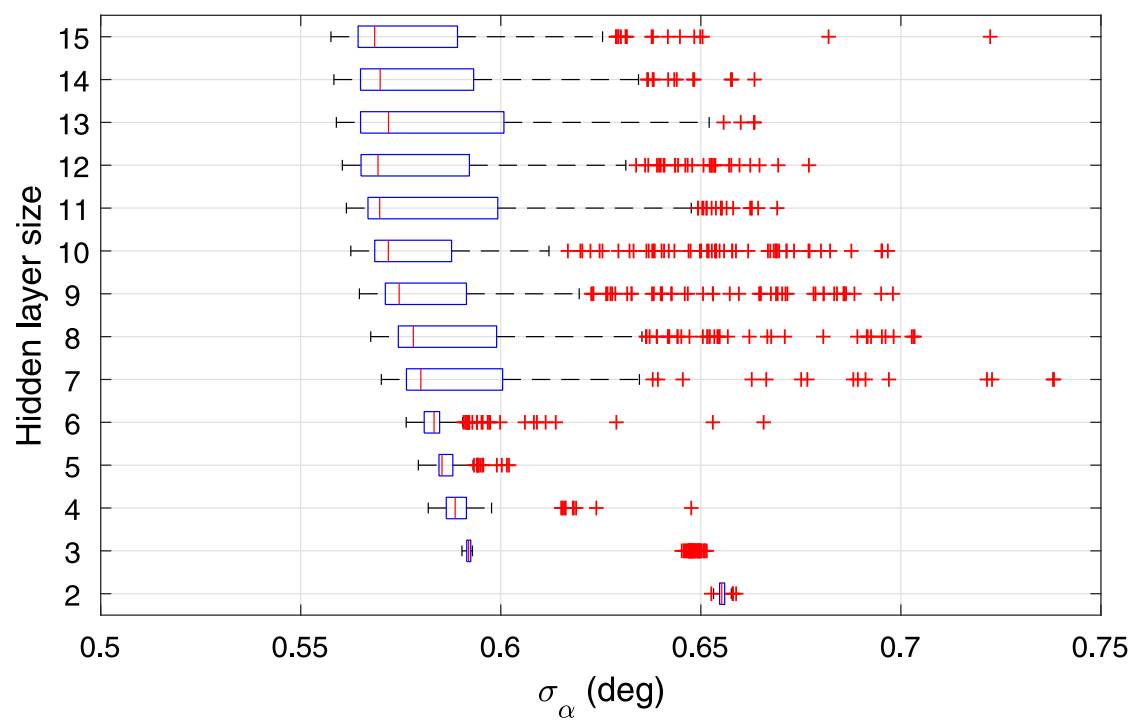

FIG. 6. Results for the 250 networks per hidden-layer size that were trained.

in-flight calibration was approximately $0.56^{\circ}$, while the 25 th percentile is at $0.58^{\circ}$. This shows that the best-case results are likely not a fluke. For the results for CFD-1, the best results for $\sigma_{\alpha}$ and $\sigma_{\beta}$ are about $0.80^{\circ}$ and $1.3^{\circ}$, respectively, while the 25 th percentiles are at $1.60^{\circ}$ and $1.80^{\circ}$. This larger gap between the best case and 25 th percentile shows that achieving results similar to the best case is less likely with CFD-1 than with in-flight
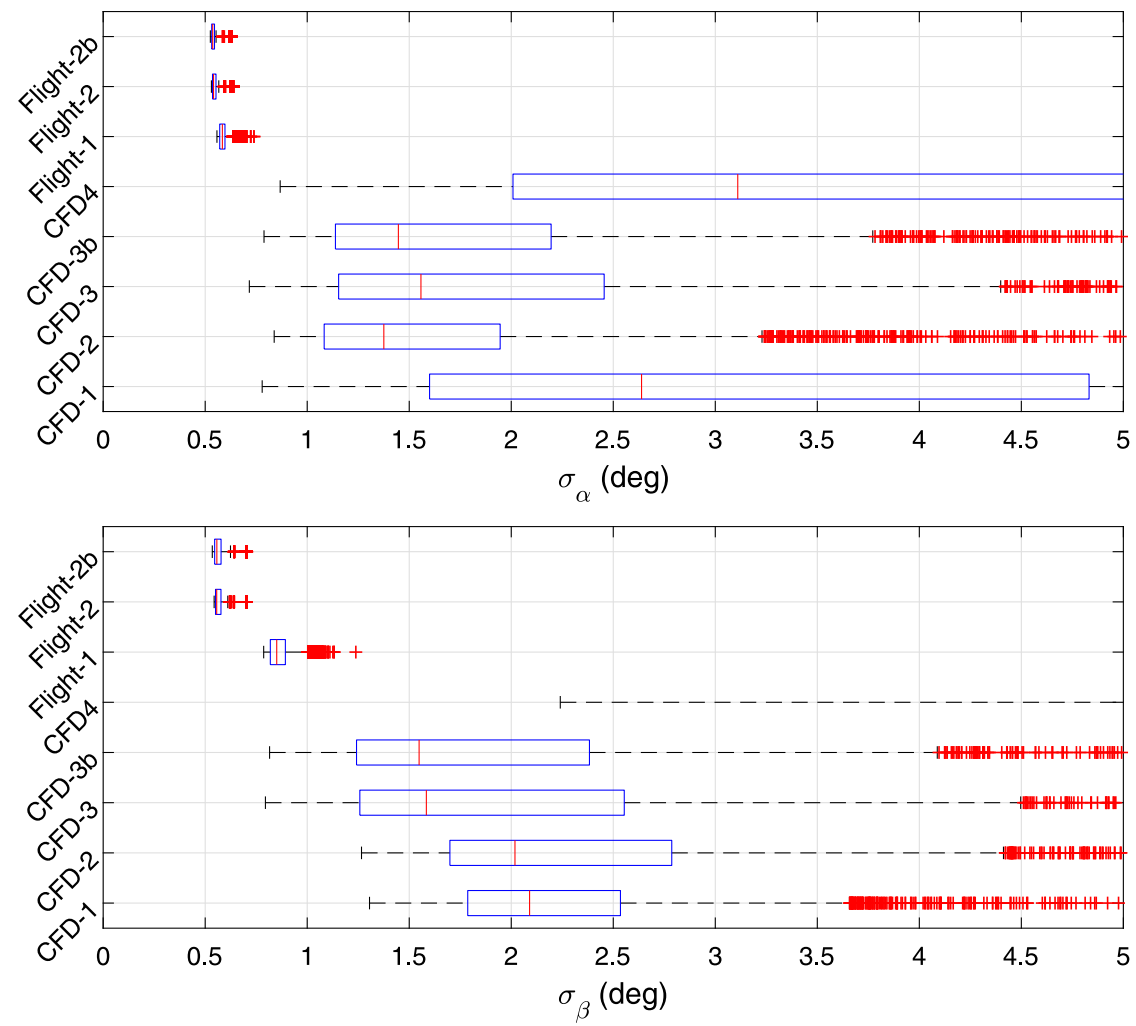

FIG. 7. Results from networks trained with Bayesian regularization and output of both (top) $\alpha$ and (bottom) $\beta$. Each method trained 250 networks using 14 different hidden-layer sizes, for a total of 3500 networks. 
TABLE 3. Summary of calibration approaches.

\begin{tabular}{ll}
\hline \hline \multicolumn{1}{c}{ Approach } & \\
\hline In-flight calibration & Inputs: $\left[\Delta P_{1-12}, \mathrm{IAS}, P_{\infty}, \rho_{\infty}\right]$ \\
& Outputs: $[\alpha, \beta]$ \\
& Calibrated with a calibration subset of the in-flight dataset; trained with Bayesian regularization \\
& Inputs: $\left[\Delta P_{1-12}, q_{\infty}, P_{\infty}, \rho_{\infty}\right]$ \\
& Outputs: $[\alpha, \beta]$ \\
& Calibrated with original CFD simulation data; trained with Bayesian regularization or \\
& Levenberg-Marquardt \\
& Inputs: $\left[\Delta P_{1-12}, q_{\infty}, P_{\infty}, \rho_{\infty}\right]$ \\
& Outputs: $[\alpha, \beta]$ \\
& Calibrated with updated CFD simulation data; trained with Bayesian regularization or Levenberg- \\
CFD-2 & Marquardt \\
& Inputs: $\left[\Delta P_{1-12}, q_{\infty}, P_{\mathrm{S} 1}, P_{\mathrm{S} 2}\right]$ \\
& Outputs: $[\alpha, \beta]$ or $\left[\alpha, \beta, q_{\infty}, P_{\infty}, \rho_{\infty}\right]$ \\
& Calibrated with updated CFD simulation data; trained with Bayesian regularization; density is an \\
CFD-3 & additional input for "b" designation \\
& Inputs: $\left[\Delta P_{1-12}\right]$ \\
& Outputs: $[\alpha, \beta]$ or $\left[\alpha, \beta, q_{\infty}, P_{\infty}, \rho_{\infty}\right]$ \\
& Calibrated with updated CFD simulation data; trained with Bayesian regularization \\
& Inputs: $\left[\Delta P_{1-12}, q_{\infty}, P_{\mathrm{S} 1}, P_{\mathrm{S} 2}\right]$ \\
CFD-4 & Outputs: $[\alpha, \beta]$ \\
& Calibrated with calibration subset of in-flight dataset; trained with Bayesian regularization; density \\
& is an additional input for "b" designation \\
\hline
\end{tabular}

calibration. Additionally, the trend of being able to more accurately estimate $\alpha$ continues, although, while $\alpha$ can be more accurate than $\beta$, the spread is much smaller with $\beta$. The 25 th percentile represents 875 networks of the 3500 networks (250 networks with 14 different hidden-layer sizes).

It is important to note that, unlike the in-flight calibration, the accuracy of the system does depend on the hidden-layer size chosen. Results from hidden-layer sizes of $8-15$ are very similar, but sizes of 7 and below are noticeably less accurate. Even though the spread may be large, with intelligent choosing of the hiddenlayer size, the spread can be reduced dramatically.

\section{c. CFD-2 calibration}

The CFD-2 approach is similar to CFD-1, with the difference being that CFD-2 uses the updated CFD simulation data. The differences between the CFD simulations are shown in Table 1. The updated CFD dataset has 588 data points as compared with 486 for CFD-1 and 24262 for the in-flight calibration dataset. Results using the original network parameters (nine nodes and sorted by best ||RMSE |) are shown in Table 2. Relative to CFD-1, the network from CFD-2 has a considerably smaller bias in the errors. In fact, the mean errors in $\alpha$ for the full dataset are less than $1 / 3^{\circ}$. When viewing the standard deviation of errors, improvements relative to CFD- 1 are seen in $\sigma_{\alpha}$ and $\sigma_{\beta}$.

The overall results are available in Fig. 7 . The bestcase results using the updated CFD simulations are close to the best-case results using the original CFD simulations. This is a very interesting result in that the best-case $\sigma$ values do not depend strongly on which CFD simulations are used. This is likely due to the fact that the networks have been trained using Bayesian regularization, which is designed to be generalizable. ${ }^{7}$ However, when looking at the 25 th percentile, CFD-2 is more accurate. The spread in smaller with the CFD-2 method, especially when looking at $\alpha$. This shows that while the best-case result is largely independent of the CFD dataset, the updated CFD dataset is more likely to produce a more accurate network.

More networks were trained, with the same range of hidden-layer sizes and number of networks, using both the original and updated CFD simulations. However, rather than use Bayesian regularization, the training method was Levenberg-Marquardt (Hagan and Menhaj 1994; Marquardt 1963). Training is faster with Levenberg-Marquardt, and it typically offers comparable accuracy to Bayesian regularization, but only for the data with which the network was trained. LevenbergMarquardt was not designed to generalize well, so it is expected that it will perform worse when trained with the original CFD dataset, because the flight dataset contains some flow conditions that are outside the bounds of the original CFD simulations. Results comparing the

\footnotetext{
${ }^{7}$ Generalizability is the ability to achieve comparable results between the data used to train the network and novel data.
} 

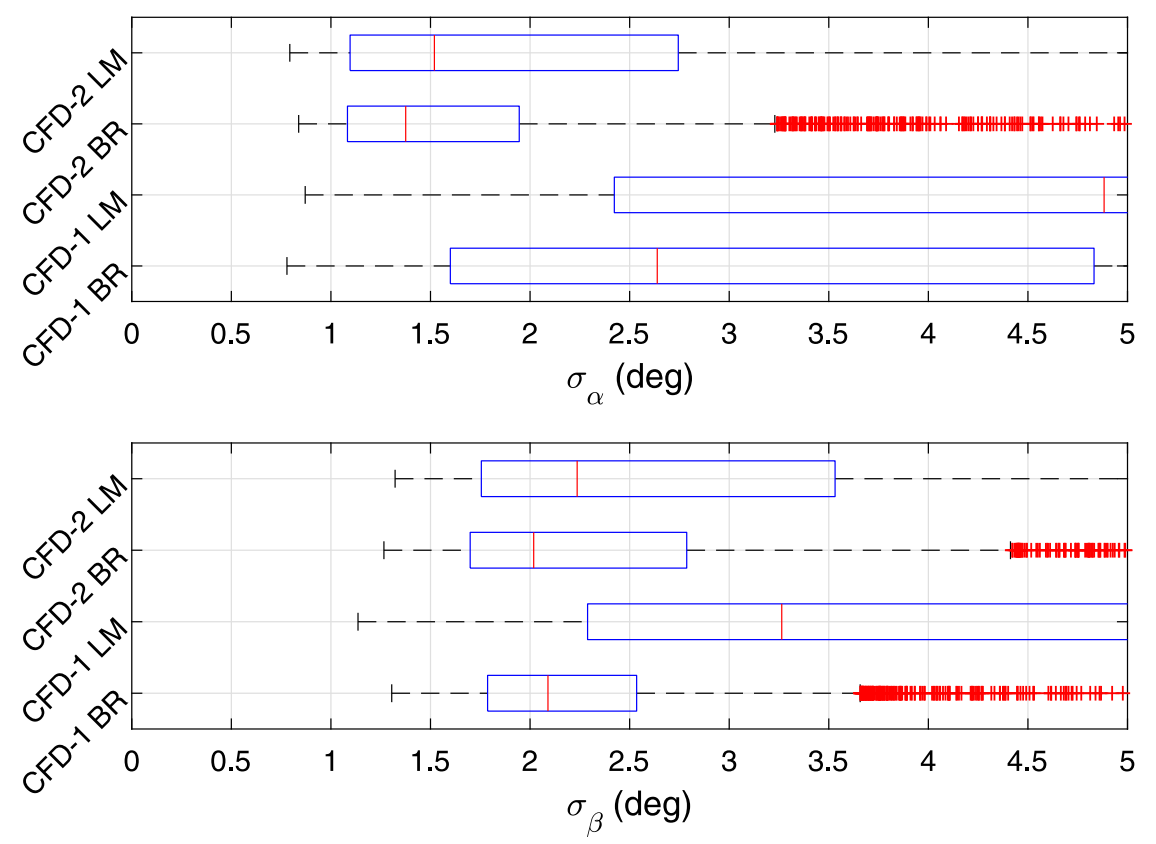

FIG. 8. Comparison of the Bayesian regularization and Levenberg-Marquardt training methods.

two training methods are shown in Fig. 8. Focusing on the updated CFD simulations of CFD-2 first, the results from Bayesian regularization and LevenbergMarquardt are similar through the 25 th percentile. When using the updated simulations, the training method is of minor importance. However, when using the original simulations (CFD-1), the training method is important. For $\alpha$, Bayesian regularization is the more accurate method, as predicted; for $\beta$, the best-case results of Levenberg-Marquardt are more accurate, but the 25th percentile is more accurate using Bayesian regularization. For all cases, the spread in the network accuracy is smaller when using Bayesian regularization. Thus, one way to improve results is to use CFD simulations that more closely match the flight conditions. A way to improve performance when the CFD simulations do not closely match the flight conditions is to use Bayesian regularization instead of Levenberg-Marquardt.

\section{d. CFD-3 calibration}

Up to this point, all of the networks trained have used the $\Delta P$ measurements from the 12 usable wing/winglet ports, $q_{\infty}, P_{\infty}$, and $\rho_{\infty}$. CFD-3 still uses the $12 \Delta P$ measurements and $q_{\infty}$, but $P_{\infty}$ and $\rho_{\infty}$ are replaced by the pressure measurements at the two fuselage static ports, $P_{\mathrm{S} 1}$ and $P_{\mathrm{S} 2}$. The switch to using $P_{\mathrm{S} 1}$ and $P_{\mathrm{S} 2}$ instead of $P_{\infty}$ provides more data and should improve the results. The removal of $\rho_{\infty}$ will make sense shortly. Outputs are still only $\alpha$ and $\beta$.
The same range of hidden-layer sizes was tested, with 250 networks per size again. Results are shown in Fig. 7. Relative to CFD-2, the best-case results for $\alpha$ are marginally better (improvement of approximately $0.10^{\circ}$ ), while the 25th percentile is identical. Although there was only minimal improvement in $\alpha$, there is a much larger improvement in the $\beta$ estimates, of approximately $0.50^{\circ}$ for both the best case and the 25th percentile. This improvement puts the best-case $\beta$ results on par with the inflight calibration results, although the 25 th percentile is approximately $0.80^{\circ}$ higher. The improvement in $\beta$ is hypothesized to be related to using data from both static ports, instead of just the average of them. The accuracy of the CFD-3 network does depend on the hidden-layer size, so choosing appropriate hiddenlayer sizes will decrease the gap between the 25th percentile of in-flight calibration and CFD-3. This is also the first approach where the system performs equally well estimating both $\alpha$ and $\beta$.

As stated previously, the fuselage stagnation and static ports, along with a temperature measure, can be used to directly calculate $q_{\infty}$ (and therefore IAS), $P_{\infty}$, and $\rho_{\infty}$ (and therefore TAS when combined with $q_{\infty}$ ). However, it should also be possible to calculate these parameters from the neural networks. To test this, networks were retrained, but with the outputs set to include $q_{\infty}, P_{\infty}$, and $\rho_{\infty}$. The reader will note that $q_{\infty}$ is now both an input and an output. The $q_{\infty}$ input is measured from the FADS, whereas the $q_{\infty}$ used as 

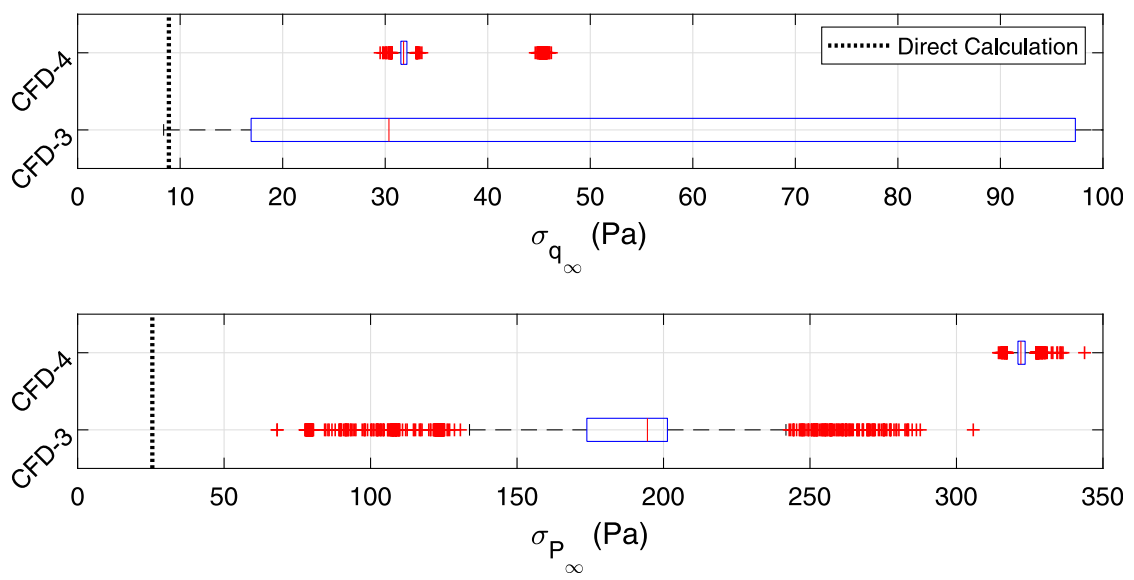

FIG. 9. Comparison of (top) $q_{\infty}$ and (bottom) $P_{\infty}$ via the direct calculation approach and both CFD-3 and CFD-4.

target data during training actually comes from the CFD simulations. This is an attempt to supply a $q_{\infty}$ measurement from the FADS and have the neural network improve the accuracy of the measurement. The only measurement of $\rho_{\infty}$ available with the flight data comes from the FADS, so having $\rho_{\infty}$ be both an input and an output would do nothing to improve the accuracy.

As previously mentioned, $P_{\infty}$ is the average of the pressure measurements at the two fuselage static port locations, $q_{\infty}$ is the pressure measurement at the fuselage stagnation port minus $P_{\infty}$, and $\rho_{\infty}$ uses the ideal gas law with $P_{\infty}$ and temperature from the FADS sensors. This direct calculation is compared with $q_{\infty}$ and $P_{\infty}$ from the MHP, with results presented as the dotted vertical line in Fig. 9. Results from CFD-3 are also shown in Fig. 9. As compared with the direct calculation, using neural networks offers no real accuracy advantage for $q_{\infty}$, and outside the best networks CFD-3 is actually worse. Estimates of $P_{\infty}$ are even less accurate. Therefore, while it is indeed possible to estimate $q_{\infty}$ and $P_{\infty}$ from neural networks trained with only CFD data, results are much better using static and stagnation ports on the fuselage with the direct calculation approach.

Results comparing the estimate of density from the neural networks to the measured density are found in Fig. 10. In flight, the density ranged from a minimum of 0.958 to a maximum of $0.998 \mathrm{~kg} \mathrm{~m}^{-3}$. While not a large range of densities, the $\sigma$ values in Fig. 10 are about $15 \%$ of the density range. One other thing to note is how consistent the different networks are. It made hardly any difference what network size, or what network within the ensemble, was chosen. While there was only a small range of densities, this shows that it may be possible to estimate density, and subsequently temperature, from only the pressure measurements. Future flight tests need to include another independent instrument that can be used to measure density, and flights should also ensure a wider range in density is experienced.

\section{e. CFD-4 calibration}

Up to this point, all of the calibration approaches somehow utilized data from the fuselage ports, whether it was the raw pressure measurements such as $P_{\mathrm{S} 1}$ or $P_{\mathrm{S} 2}$, or whether the fuselage ports were used for a direct calculation of static or dynamic pressure. CFD-4 will instead ignore data from the three fuselage ports. This will help to determine the importance of the fuselage ports on the accuracy of the FADS and to answer whether a flush air data system can be designed without typical stagnation and static ports. The updated CFD simulations will be used for training, but the inputs will only be the $12 \Delta P$ measurements from the wing/winglet sensors.

Overall results from the different hidden-layer sizes are presented in Fig. 7. The best-case $\alpha$ results are worse than all of the other calibration approaches but are still within $0.1^{\circ}$ of all other approaches. However, the spread of the results is far wider with CFD-4 than any other method. This is even more true when viewing the $\beta$ results. In fact, the 25 th percentile for $\beta$ is not even within the scale of the plot. For $\beta$, the 25 th percentile is at $5.7^{\circ}$, the median is at $8.9^{\circ}$, the 75 th percentile is $14.5^{\circ}$, and the outliers start at $27.7^{\circ}$. For $\alpha$, the median is shown, but the 75 th percentile is off the chart at $5.2^{\circ}$, and the outliers start at $9.9^{\circ}$.

Both CFD-1 and CFD-2 average the two static ports together for a single static pressure reading that is an input into the networks; CFD-3 uses both static port measurements, and experiences an increase in $\beta$ accuracy; 


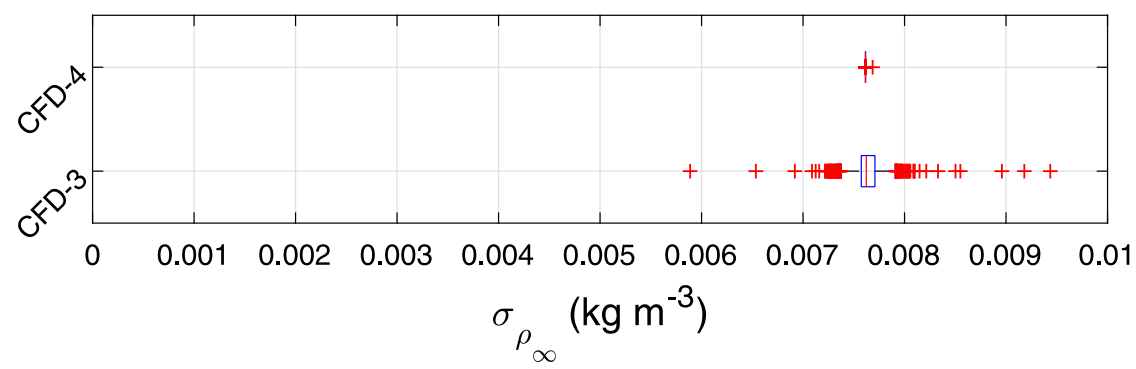

FIG. 10. Density estimates using CFD trained networks.

CFD-4 ignores the static ports entirely (along with the stagnation port) and has the worst $\beta$ accuracy. This result highlights the apparent importance the fuselage static port measurements have on the $\beta$ results. It is interesting to note that changing how the static ports are used (and whether they are used at all) has a significantly smaller effect on the $\alpha$ results.

Estimates of $q_{\infty}$ and $P_{\infty}$ for the CFD-4 approach are displayed in Fig. 9. It is immediately clear that both $q_{\infty}$ and $P_{\infty}$ are significantly less accurate than any other approach to estimating those quantities so far. At the mean dynamic pressure of $250 \mathrm{~Pa}$ during flight tests, the $1-\sigma$ error of approximately $30 \mathrm{~Pa}$ translates to an error of about $1.2 \mathrm{~m} \mathrm{~s}^{-1}$ for indicated airspeed. The $1-\sigma$ error of $325 \mathrm{~Pa}$ is $0.4 \%$ of the mean static pressure during flight, but is nearly $20 \%$ of the range of static pressures measured. Clearly, using only the wing/winglets ports greatly reduces the accuracy of the network estimates for $q_{\infty}$ and $P_{\infty}$. Density, on the other hand, is only minimally less accurate when data from the fuselage ports are ignored, as shown in Fig. 10.

\section{Improvements and discussion}

It is clear that using both static ports with the neural networks improves the accuracy, with $\beta$ seeing a greater improvement than $\alpha$. To further test the effects of both static ports, the in-flight calibration approach was tweaked so that instead of using IAS, $P_{\infty}$, and $\rho_{\infty}$ as inputs, $q_{\infty}, P_{\mathrm{S} 1}$, and $P_{\mathrm{S} 2}$ were used, just like the CFD-3 approach. This approach is labeled as in-flight calibration 2 in Fig. 7. Relative to the original in-flight calibration, $\alpha$ is marginally more accurate now, but $\beta$ experienced a greater improvement. In fact, $\alpha$ and $\beta$ results are now on par with each other, whereas in every other case, $\alpha$ has been more accurate than $\beta$. This further underscores the importance of utilizing as much data from all of the ports as possible.

The reader will notice that some of the calibration approaches utilized density measurements, while others did not. In-flight calibration $2 b$ and CFD- $3 b$ are attempts to discern the importance of density measurements on the accuracy of the FADS. These two calibration approaches are identical to their respective originals, but with the addition of density as an input. The results from in-flight calibration 2 and in-flight calibration $2 b$ are virtually identical, with in-flight calibration $2 b$ having a slightly smaller spread. Results from CFD-3 and CFD-3b are also nearly identical to each other, with differences of only a few hundredths of a degree for both $\alpha$ and $\beta$. This shows that adding density as an input to the FADS, when the inputs already include $q_{\infty}, P_{\mathrm{S} 1}$, and $P_{\mathrm{S} 2}$, only marginally increases the accuracy of the system.

For this project, it was assumed that the port locations on the physical aircraft perfectly matched the intended locations found in Laurence and Argrow (2018) and Fig. 4. In reality, the actual port locations on the aircraft were likely installed close to, but not exactly matching, the intended locations. This discrepancy between the actual and intended installation locations is due to the installation being performed by hand, and the associated errors. Thus, the locations from the CFD simulations used for calibration are unlikely to perfectly match the actual installation locations, leading to some errors. A 3D scan of the physical aircraft with the installed ports would lead to a decrease in the port position uncertainty. Alternatively, some form of computer-controlled installation (e.g., 3D printing) could also decrease the uncertainty in the actual port locations. Either approach would lead to a better match between port locations from CFD and the true port locations, and thus an expected increase in accuracy for the CFD calibrations.

\section{Conclusions}

A numerical approach to calibrating flush air data systems has been presented and analyzed. As opposed to training neural networks on experimental data, as has been previously reported, the networks were trained with data derived from computational fluid dynamics simulations. Multiple approaches utilizing the CFD data were explored, including using the original 
CFD data, using updated CFD data that better matches the flight conditions, using data from both fuselage static pressure ports instead of their average, and even ignoring the fuselage ports entirely.

Unsurprisingly, calibrating with the experimental data was always better than calibrating with CFD data, though the CFD calibration was able to achieve results within approximately $1 / 3^{\circ}$ of the flight calibration. Matching the CFD simulations to the flight conditions improves the accuracy, but if that is not possible, using a more general training method, such as Bayesian regularization, improves the results. Other air data parameters, such as dynamic and static pressure, can be estimated with neural networks, but the direct approach is more accurate. It also appears possible to estimate density simply from the pressure measurements, but more testing is required to confirm this and truly assess the accuracy. Data from the fuselage ports play a small role in the angle of attack results, but significantly affect the angle of sideslip results. Using pressure from both fuselage static ports, instead of a single average static pressure value, increases the FADS accuracy, with sideslip being the biggest beneficiary. This is also true when using the flight data for calibration, and results in the angle of attack and sideslip having a nearly identical accuracy. It was also shown that using density as an input has no effect when already using $q_{\infty}, P_{\mathrm{S} 1}$, and $P_{\mathrm{S} 2}$ as inputs. It is assumed that greater accuracy will be achieved when the CFD cells used more closely match the actual installation locations, but more testing is required to characterize this relationship.

Future work should focus on determining the exact pressure port locations, either through 3D scanning of the aircraft after installation, or through improved installation techniques, such as using $3 \mathrm{D}$ printing for the ports. Not explored in this paper, but a potentially useful approach would be a hybrid CFD and flight data method, where CFD simulations are used to augment reduced flight testing. This would not eliminate field calibration like an exclusive CFD approach, but could reduce the required amount of flight testing. While not as accurate as calibration with flight data, CFD calibration of a flush air data system was shown to be a viable alternative, and it eliminates the need for costly and time-consuming field calibrations.

Acknowledgments. This material is based upon work supported by the National Science Foundation under Grant IIS-1527919. The authors also acknowledge the help of Abhilash Manjunath for designing the code to collect the MS8607 sensor data; Thomas Lillis for implementing the data logging software on the aircraft; Dan Hesselius for piloting the Skywalker and general hardware support; Steve Borenstein for the circuit board design and sensor support; Chris Choate, Chris Brown, and Will Finamore for aircraft support; and the rest of the RECUV/IRISS support team.

\section{REFERENCES}

Aeroprobe, 2016: Aeroprobe micro air data system user manual: Revision D. 2nd ed. Aeroprobe Corporation Rep., 38 pp.

Callegari, S., M. Zagnoni, A. Golfarelli, M. Tartagni, A. Talamelli, P. Proli, and A. Rossetti, 2006: Experiments on aircraft flight parameter detection by on-skin sensors. Sens. Actuators, 130A-131A, 155-165, https://doi.org/10.1016/j.sna.2005.12.026.

Cary, J. P., and E. R. Keener, 1965: Flight evaluation of the X-15 ball-nose flow-direction sensor as an air-data system. NASA Tech. Note D-2923, 21 pp.

Cobleigh, B., S. Whitmore, E. Haering Jr., J. Borrer, and V. Roback, 1999: Flush airdata sensing (FADS) system calibration procedures and results for blunt forebodies. Ninth Int. Space Planes and Hypersonic Systems and Technologies Conf., Norfolk, VA, American Institute of Aeronautics and Astronautics, 99-4816, https://doi.org/10.2514/ 6.1999-4816.

COESA, 1976: U.S. Standard Atmosphere, 1976. NOAA, 227 pp.

Crowther, W. J., and P. J. Lamont, 2000: A neural network approach to the calibration of a flush air data system. University of Manchester School of Engineering Tech. Rep., 21 pp.

Elston, J., B. Argrow, M. Stachura, D. Weibel, D. Lawrence, and D. Pope, 2015: Overview of small fixed-wing unmanned aircraft for meteorological sampling. J. Atmos. Oceanic Technol., 32, 97-115, https://doi.org/10.1175/JTECH-D-13-00236.1.

Fei, H., R. Zhu, Z. Zhou, and J. Wang, 2007: Aircraft flight parameter detection based on a neural network using multiple hot-film flow speed sensors. Smart Mater. Struct., 16, 12391245, https://doi.org/10.1088/0964-1726/16/4/035.

Foresee, F. D., and M. T. Hagan, 1997: Gauss-Newton approximation to Bayesian regularization. Proc. Int. Joint Conf. on Neural Networks, Houston, TX, IEEE, 1930-1935, https:// doi.org/10.1109/ICNN.1997.614194.

Haering, E. A., 1995: Airdata measurement and calibration. NASA Tech. Memo. 104316, 19 pp., https://www.nasa.gov/centers/ dryden/pdf/88377main_H-2044.pdf.

Hagan, M. T., and M. B. Menhaj, 1994: Training feedforward networks with the Marquardt algorithm. IEEE Trans. Neural Networks, 5, 989-993, https://doi.org/10.1109/72.329697.

Hornik, K., M. Stinchcombe, and H. White, 1989: Multilayer feedforward networks are universal approximators. Neural Networks, 2, 359-366, https://doi.org/10.1016/0893-6080(89) 90020-8.

Houston, A., R. Laurence, T. Nichols, S. Waugh, B. Argrow, and C. Ziegler, 2016: Intercomparison of unmanned aircraftborne and mobile mesonet atmospheric sensors. J. Atmos. Oceanic Technol., 33, 1569-1582, https://doi.org/10.1175/JTECH-D15-0178.1.

Johnston, I. A., P. A. Jacobs, and T. Shimoda, 1998: Flush air data system calibration using numerical simulation. J. Spacecr. Rockets, 35, 812-820, https://doi.org/10.2514/2.3404.

Kalogiros, J. A., and Q. Wang, 2002: Calibration of a radomedifferential GPS system on a Twin Otter research aircraft for turbulence measurements. J. Atmos. Oceanic Technol., 19, 159-171, https://doi.org/10.1175/1520-0426(2002)019<0159: COARDG $>2.0 . \mathrm{CO} ; 2$. 
Karlgaard, C. D., P. Kutty, M. Schoenenberger, M. M. Munk, A. Little, C. A. Kuhl, and J. Shidner, 2014: Mars Science Laboratory entry atmospheric data system trajectory and atmosphere reconstruction. J. Spacecr. Rockets, 51, 1029-1047, https://doi.org/10.2514/1.A32770.

Kocer, G., M. Mansour, N. Chokani, R. Abhari, and M. Müller, 2011: Full-scale wind turbine near-wake measurements using an instrumented uninhabited aerial vehicle. J. Sol. Energy Eng., 133, 041011, https://doi.org/10.1115/1.4004707.

Larson, T. J., S. A. Whitmore, L. J. Ehernberger, J. B. Johnson, and P. M. Siemers III, 1987: Qualitative evaluation of a flush air data system at transonic speeds and high angles of attack. NASA Tech. Paper 2716, 64 pp.

Laurence, R. J., III, and B. M. Argrow, 2018: Development and flight test results of a small UAS distributed flush airdata system. J. Atmos. Oceanic Technol., 35, 1127-1140, https:// doi.org/10.1175/JTECH-D-17-0192.1.

Lie, F. A. P., and D. Gebre-Egziabher, 2013: Synthetic air data system. J. Aircr., 50, 1234-1249, https://doi.org/10.2514/1.C032177.

MacKay, D. J. C., 1992: Bayesian interpolation. Neural Comput., 4, 415-447, https://doi.org/10.1162/neco.1992.4.3.415.

Marquardt, D. W., 1963: An algorithm for least-squares estimation of nonlinear parameters. J. Soc. Ind. Appl. Math., 11, 431-441, https://doi.org/10.1137/0111030.

MathWorks, 2017: initnw: Nguyen-Widrow layer initialization function. MathWorks, accessed 19 June 2017, https:// www.mathworks.com/help/nnet/ref/initnw.html.

__ 2018a: purelin: Linear transfer function. MathWorks, accessed 16 May 2018, https://www.mathworks.com/help/nnet/ ref/purelin.html.

_ 2018b: tansig: Hyperbolic tangent sigmoid transfer function. MathWorks, accessed 16 May 2018, https://www.mathworks.com/ help/nnet/ref/tansig.html.

Mayer, S., and G. Hattenberger, 2012: A "no-flow-sensor" wind estimation algorithm for unmanned aerial systems. Int. J. Micro Air Veh., 4, 15-29, https://doi.org/10.1260/1756-8293.4.1.15.

Mohammadi, B., and O. Pironneau, 1994: Analysis of the K-Epsilon Turbulence Model. John Wiley and Sons, $194 \mathrm{pp}$.

Nguyen, D., and B. Widrow, 1990: Improving the learning speed of 2-layer neural networks by choosing initial values of the adaptive weights. Int. Joint Conf. on Neural Networks, San Diego, CA, IEEE, 21-26, https://doi.org/10.1109/IJCNN.1990.137819.

Pruett, C. D., H. Wolf, M. L. Heck, and P. M. Siemers III, 1983: Innovative air data system for the space shuttle orbiter. J. Spacecr. Rockets, 20,61-69, https://doi.org/10.2514/3.28357.

Quindlen, J. F., and J. W. Langelaan, 2013: Flush air data sensing for soaring-capable UAVs. 51st Aerospace Sciences Meeting including the New Horizons Forum and Aerospace Exposition, Grapevine, TX, American Institute of Aeronautics and Astronautics, 2013-1153, https://doi.org/10.2514/6.2013-1153.
Razzaqi, S. A., C. Bode, T. Eggers, and M. K. Smart, 2012: Development of functional relationships for air-data estimation using numerical simulations. 18th Australasian Fluid Mechanics Conf., Launceston, Australia, Australasian Fluid Mechanics Society.

Rohloff, T. J., 1998: Air data sensing from surface pressure measurements using a neural network method. AIAA J., 36, 2094 2101, https://doi.org/10.2514/2.312.

Samy, I., I. Postlethwaite, D. Gu, and J. Green, 2010: Neuralnetwork-based flush air data sensing system demonstrated on a mini air vehicle. J. Aircr., 47, 18-31, https://doi.org/ 10.2514/1.44157.

Siemens, 2017: STAR-CCM+. Siemens, accessed 31 March 2017, http://mdx.plm.automation.siemens.com/star-ccm-plus.

Siemers, P., H. Wolf, and P. F. Flanagan, 1983: Shuttle entry air data system concepts applied to space shuttle orbiter flight pressure data to determine air data-STS 1-4. 21st Aerospace Sciences Meeting, Reno, NV, American Institute of Aeronautics and Astronautics, 1-12, https://doi.org/10.2514/6.1983-118.

Srivastava, A., A. J. Meade, and A. A. Mokhtarzadeh, 2010: A hybrid data-model fusion approach to calibrate a flush air data sensing system.Infotech@Aerospace, Atlanta, GA, American Institute of Aeronautics and Astronautics, I@A-17, https:// doi.org/10.2514/6.2010-3347.

TE Connectivity, 2015: PTH combination digital sensor: MS860702BA01. TE Connectivity, http://www.te.com/usa-en/productCAT-BLPS0018.html.

Telionis, D., Y. Yang, and O. Rediniotis, 2009: Recent developments in multi-hole probe (MHP) technology. 20th Int. Congress of Mechanical Engineering, Gramado, Brazil, Associação Brasileira de Engenharia e Ciências Mecânicas, 29 pp.

van den Kroonenberg, A., T. Martin, M. Buschmann, J. Bange, and P. Vorsmann, 2008: Measuring the wind vector using the autonomous mini aerial vehicle M2AV. J. Atmos. Oceanic Technol., 25, 1969-1982, https://doi.org/10.1175/2008JTECHA1114.1.

Whitmore, S. A., 1991: Development of a pneumatic high-angle-ofattack flush airdata sensing (HI-FADS) system. NASA Tech. Memo. 104241, 30 pp.

— R. J. Davis, and J. Fife, 1996: In-flight demonstration of a real-time flush airdata sensing system. J. Aircr., 33, 970-977, https://doi.org/10.2514/3.47043.

Wildmann, N., M. Hofsäß, F. Weimer, A. Joos, and J. Bange, 2014a: MASC - A small remotely piloted aircraft (RPA) for wind energy research. Adv. Sci. Res., 11, 55-61, https://doi.org/ 10.5194/asr-11-55-2014.

_ S. Ravi, and J. Bange, 2014b: Towards higher accuracy and better frequency response with standard multi-hole probes in turbulence measurement with remotely piloted aircraft (RPA). Atmos. Meas. Tech., 7, 1027-1041, https://doi.org/ 10.5194/amt-7-1027-2014. 